\title{
Outcome of conservative surgical treatment of deep infiltrating endometriosis
}

\author{
Sylvie Gordts • Patrick Puttemans • Rudi Campo • \\ Marion Valkenburg $\cdot$ Stephan Gordts
}

Received: 13 December 2011 / Accepted: 8 August 2012 /Published online: 18 September 2012

(C) Springer-Verlag 2012

\begin{abstract}
Deep infiltrating endometriosis, which is frequently associated with pain, is diagnosed at clinical examination and with indirect imaging techniques like ultrasound and MRI. The aim of this study was to evaluate complications and recurrence rate after laparoscopic resection of deep infiltrating endometriosis by shaving technique. Between January 2004 and December 2010, 74 procedures for deep infiltrating endometriosis were performed in patients with pain and/or infertility. The endometriotic plaques were resected laparoscopically using scissors and bipolar and/or unipolar current. If rectosigmoidal invasion was present, a shaving was performed. Mean age was 31.7 years $(\mathrm{SD} \pm 4.4)$. The vaginal nodule was a solitary lesion in $4 \%$ of the patients without involvement of the ovaries, rectum or bladder. In $86 \%$ of the cases, the rectosigmoid was involved as well. Mean follow-up was 776 days $(\mathrm{SD}=$ 465). One patient developed postoperatively a severe complication with intestinal perforation secondary to thermal necrosis $(1.4 \%)$. In four patients recurrence of symptoms was noted $(8 \%)$. Conservative surgery for deep infiltrating endometriosis resulted in the relief of pain, with a low postoperative complication rate $(1.4 \%)$. This shaving technique also resulted in a limited risk of recurrence of the symptoms $(8 \%)$.
\end{abstract}

Keywords Deep endometriosis · Surgery $\cdot$ Outcome

S. Gordts $(\bowtie) \cdot$ P. Puttemans $\cdot$ R. Campo $\cdot$ M. Valkenburg $\cdot$

S. Gordts

LIFE,

Tiensevest 168,

Leuven 3000, Belgium

e-mail: Sylvie.Gordts@lifeleuven.be

\section{Background}

Endometriosis is characterized by the presence of endometrial glands and stroma outside the uterine cavity. It primarily affects women of fertile age, and represents a relevant clinical issue as it causes pain $[1,2]$ and infertility $[2,3]$. Rectovaginal endometriosis (RVE) consists of an endometriotic nodule within the connective tissue between the anterior rectal wall and the vagina. The incidence of bowel infiltration among women with endometriosis varies between 6 and $12 \%$ [4]. Deep endometriosis involving the bowel is most frequently localised in the rectovaginal septum $[4,5]$ and less in the sigmoid. Other bowel localisations are relatively rare [6].

Surgery is the best therapeutic option for symptomatic rectovaginal endometriosis. Several surgical techniques have been proposed with no consensus existing. Different procedures have been described in terms of parameters such as the size of the nodule and the affected bowel circumference, depth of lesions on the rectal wall, presence of other endometriotic foci and experience of the surgeon [7-9]. The most common surgical procedures used in the treatment of RVE are the following: superficial thickness excision (shaving), resection of the nodule with excision of the anterior side of the rectum (discoid resection) and colorectal segmental resection. At present, some surgeons prefer colorectal resection, as they are convinced that this is the treatment of choice to improve pain and to avoid relapses [10-13]. Other groups prefer the excision of the endometriotic nodule, since the rate of morbidity is lower and the recurrence of pain is similar $[14,15]$. The choice for colorectal resection is supported by studies showing that microscopic endometriotic lesions usually exist around the main rectal nodule [16, 17]. But the clinical implication of leaving microscopic foci of endometriosis on the digestive tract remains unknown [18]. The aim of this study was to 
evaluate complications and recurrence rate after laparoscopic resection of deep infiltrating endometriosis by the shaving technique.

\section{Materials and methods}

Between January 2004 and December 2010, 74 procedures for deep infiltrating endometriosis were performed in patients with pain and/or infertility. Fifteen patients presented with pain symptoms, without fertility problems; 57 patients presented with pain and fertility problems, while two patients had only fertility problems and no pain (Table 1). These procedures were retrospectively reviewed for complications and relapse of symptoms.

Examination with a speculum revealed either a normal vaginal mucosa or a protruded blue nodule in the posterior fornix. By palpation, the diameter of the lesion could be evaluated. Palpation is often painful, and the presence of a nodule accounts for symptoms such as deep dyspareunia and dysmenorrhea. Preoperatively, a full bowel preparation was performed in all patients.

The endometriotic nodules were resected laparoscopically. In all cases a 5-mm laparoscope, in the umbilical position, and three 5-mm trocars were used. First, an adhesiolysis was performed. When ovarian endometriotic cysts were present, these were treated in the first step by opening the cyst and coagulation of the inner endometriotic wall using a BICAP probe (Storz). Resection of the nodule was performed using scissors and bipolar and/or unipolar current. Excision was guided visually and by tactile feedback of the indurations during the endoscopic excision.

Dissection was started by freeing the anterior rectum until the loose tissue of the rectovaginal space was reached. If rectosigmoidal invasion was present, a shaving was performed. With this technique as much as possible of the endometriotic tissue is removed without opening the intestine. This technique was performed independently of the size and depth of the lesion. A careful sharp dissection was performed until the rectum was completely free and identifiable below the lesion. Excision of the fibrotic tissue

Table 1 Number of patients with respective sites of endometriosis lesions according to symptoms

\begin{tabular}{lllll}
\hline Site of endometriosis & Pain $^{\mathrm{a}}$ & Infertility $^{\mathrm{b}}$ & Infertility + pain & Total \\
\hline Ovarian endometriosis & 4 & 2 & 31 & 37 \\
Rectosigmoid & 13 & 0 & 51 & 64 \\
Bladder & 1 & 1 & 8 & 10 \\
Total & 15 & 2 & 57 & 74 \\
\hline
\end{tabular}

${ }^{a}$ Pain symptoms without infertility

${ }^{b}$ Infertility without any symptoms of pain on the side of the rectum was performed after the rectal dissection was complete. The rectal muscle defects could be closed with suture. The lesions extending totally through the vagina were treated with en bloc laparoscopic resection of the lesion with the vaginal mucosa. In these cases the fornix posterior was opened. After complete resection of the nodule, the posterior vaginal wall was closed laparoscopically.

In order to exclude unnoticed rectum perforations, at the end of the operation, the pouch of Douglas was filled with irrigation fluid and subsequently, air was brought into the rectum. If the endometriotic nodule or fibrosis included ureter involvement, intra-operative ureter stents were used. The women were normally kept in the hospital for $36 \mathrm{~h}$ after surgery.

\section{Findings}

The mean age of the patients was 31.7 years ( $\mathrm{SD} \pm 4.4)$. The mean size of the rectovaginal nodule was $1.9 \mathrm{~cm}$. The size of the nodule was estimated by clinical examination and inspection during surgical resection of the nodule. By excision of the nodule, the posterior fornix of the vagina was opened in $42 \%$ of the cases.

The vaginal nodule was a solitary lesion in $4 \%$ of the patients without involvement of ovaries, rectum or bladder. In most cases the endometriosis was a complex disease involving different structures (sacrouterine ligaments, rectosigmoid, ureter and ovaries). In 37 cases one or both ovaries had an endometriotic cyst. In $86 \%(n=64)$ of the cases, the bowel was involved. In $14 \%$ of the patients, bladder endometriosis was present (Table 1). Thirteen patients had been operated for endometriosis in the past in other centres.

In $91 \%(n=58)$ of the patients with bowel involvement, shaving was performed. During the shaving, opening of the bowel mucosa was performed in five patients $(8 \%)$. Only on one patient a discoid resection was performed. In two patients when the appendix was involved, appendectomy was carried out. In four patients no treatment of the bowel involvement was performed since they had no bowel symptoms. In these patients only a resection of the vaginal part of the nodule was performed. In one of these four patients, a second laparoscopy with bowel shaving is performed 2 years later because of bowel symptoms.

One patient developed postoperatively a complication with intestinal perforation secondary to thermal necrosis $(1.4 \%)$. This patient presented with pain and fever 10 days postoperatively. An urgent laparoscopy was performed showing bowel perforation with necrosis. A partial bowel resection was performed. Other complications like ureteral damage, urinary retention or fistulas were not noted.

Only two patients received preoperatively GnRH analogues because of pain and waiting period before the 
Fig. 1 Pregnancy outcome. Total $=$ total of patients with follow-up of at least 1 year; male factor $=$ abnormal sperm analysis; aim for spontaneous conception $=$ no fertility treatment for at least 6 months; spontaneous pregnancy $=$ pregnancy without fertility treatment

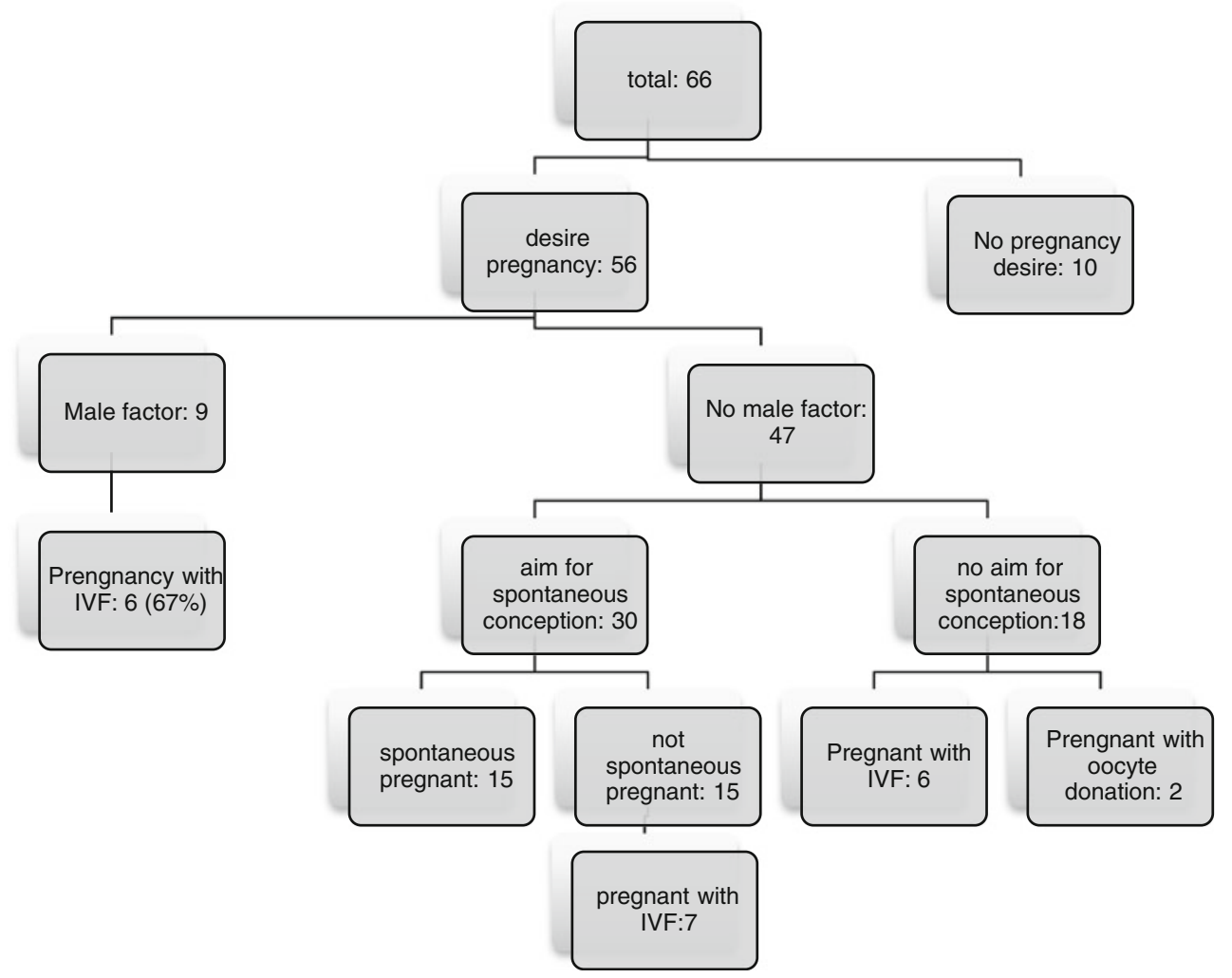

operation could be performed. Ten patients received GnRHagonist treatment for 3 months postoperatively. Six of them were given because they were referred for immediate starting of in vitro fertilization (IVF) treatment and in four patients because on MRI, adenomyosis was suspected.

Only patients with a follow-up of at least 1 year were included for further analysis. Eight patients were lost of follow-up and excluded. Mean follow-up was 776 days $(\mathrm{SD}=465)$.

In six patients recurrence of symptoms was noted (8\%). The mean time of recurrence was 540 days. In four patients an endometriotic cyst was visualised on ultrasound. In three patients a second laparoscopy was performed. Two laparoscopies showed invasion in the bowel serosa. One laparoscopy showed only recurrence of an endometriotic cyst. In one patient with a cyst on ultrasound, an aspiration of the cyst was performed. One patient had only recurrence of symptoms without obvious pathology; thus, no laparoscopy was performed.

We also evaluated the pregnancy outcome after surgery (Fig. 1). Ten patients did not try to conceive. In the rest of the group, we noted an overall pregnancy rate of $63 \%$. In nine couples a male factor was present. Those patients were immediately referred for IVF. In this group a mean of 7.7 oocytes were retrieved with a fertilization rate of $65 \%$. They reached a pregnancy rate of $67 \%$. In the group with no male factor, some patients were advised not to opt for a spontaneous pregnancy. These were older patients or patients with previous infertility treatments. They were directed towards IVF $(n=16)$ or oocyte donation $(n=2)$. In the patients for IVF treatment, a mean of six oocytes were retrieved with a fertilization rate of $61 \%$. In this group a pregnancy rate of $37 \%$ was reached. If no ovarian endometriosis was present, a mean of 7.5 oocytes were retrieved. In those patients where only one ovary had endometriosis, a mean of 7.4 oocytes could be retrieved. If both ovaries had ovarian endometriosis, a mean of eight oocytes were retrieved at oocyte pick-up. Two patients were poor responders in the IVF treatment and they started an oocyte donation treatment. The ages of these two patients were 34 and 38 years.

Thirty couples were advised to aim for a spontaneous pregnancy. The mean age of these patients was 29.7 years. The spontaneous pregnancy rate was $50 \%$ in this study. In those patients with associated ovarian endometriosis, the spontaneous pregnancy rate was also $50 \%$ (Table 2). The mean time to spontaneous conception was 7.4 months.

Table 2 Spontaneous pregnancy according to localisation of the lesion in patients with desire of pregnancy and no male pathology

\begin{tabular}{lll}
\hline Localisation of the lesion & Number $^{\mathrm{a}}$ & Spontaneous pregnancy \\
\hline Ovarian endometriosis & 16 & 8 \\
Rectosigmoid & 24 & 11 \\
Solitary vaginal & 2 & 1 \\
\hline
\end{tabular}

${ }^{\mathrm{a}}$ Infertility without any symptoms of pain 


\section{Conclusions}

Our study provides information regarding the outcome related to the shaving technique for patients with deep endometriosis. Over the last decade, it seems to have been widely accepted that complete excision of deep endometriosis is the treatment of choice. The idea is that the excision should be complete in order to achieve maximal pain relief and minimal recurrences. To our knowledge, however, there are no data to substantiate this. The decision to perform bowel resection seems to be based on attitude rather than on data. The argument used by some gynaecologists and surgeons to defend bowel resection is that this procedure is more 'radical'. But, even with bowel resection, the margins are not free of disease in more than $10 \%$ of cases [15, 19]. Other studies have shown that residual lesions in the muscularis of the rectum do not evolve and remain constant for a long time [14]. The question remains as to whether complete long-term relief of pain systematically requires complete resection of the digestive foci, when taking into consideration the risk of postoperative complications and unpleasant functional symptoms. The 'price to pay' for segmental bowel resection is presented by possible complications. It should also be pointed out that colorectal segmental resection is a complex procedure, sometimes resulting in pelvic nerve damage and unpleasant urinary and digestive symptoms $[15,20]$. A review [21] of 34 articles of bowel resections for deep endometriosis showed an overall complication rate of $22 \%$. Major complications occurred in $11 \%$ of women, that is $6.4 \%$ with severe bowel complications (leakage rate, $1.9 \%$; fistula rate, $1.8 \%$; and severe obstruction rate, $2.7 \%$ ), $2.5 \%$ with haemorrhage and $1 \%$ with infections. Minor complications occurred in $14.7 \%$ of the women, with an incidence of temporary bowel dysfunction of $3.6 \%$ and of bladder dysfunction of $8 \%$. Another review of Payá [9] concluded that when surgical treatment does not include intestinal resection, the rates of surgical complications are low, in the majority of papers not reaching $5 \%$, with bladder and intestinal dysfunction practically non-existent. They conclude that segmental bowel resection should be performed in selected cases. The indication for bowel resection might be represented by important bowel stenosis. Also in our study, low surgical complication rate of $1.4 \%$ is reached. We cannot exclude that the fact that the patients were mainly infertility patients and not pelvic pain patients, which influenced these results. The study of Fanfani et al. compared postoperative complications to the patient satisfaction and recurrence rates between two laparoscopic techniques (discoid versus segmental resection) [18]. They conclude that the complication rate is lower in the discoid resection group, but patient satisfaction and recurrence rate are comparable in both groups.
In most cases, perirectal perivisceritis may be left in place. The residual lesion in the muscularis of the rectum does not seem to evolve and remains constant for a long time. Patients are usually free of symptoms, so we can consider that systematic bowel resection in case of perirectal visceritis and rectal muscularis involvement is unnecessary. Moreover, such surgery increases morbidity and is responsible for more adhesions resulting from extensive lateral dissection. In case of bowel occlusion and rectal bleeding with rectal mucosa involvement, resection of the rectosigmoid junction can be performed.

Little data are available on the fertility and pregnancy outcome after surgery for deep infiltrating endometriosis. Some studies have shown a higher pregnancy rate for minimally invasive procedures (shaving and discoid resection) compared with segmental resection [22]. As we are a fertility unit, most of our patients present with infertility. The results of this study demonstrate that even in this subfertile population, a good spontaneous pregnancy rate can be achieved after conservative surgery. The spontaneous pregnancy rate of $50 \%$ in our study was similar as reported in other studies (Darai, Marpeau et al. 2005; Donnez and Squifflet 2010). Compared to our overall IVF population, the number of oocytes retrieved was slightly lower (7.3 versus 8.9). The fertilization rates were identical (63 versus $62.3 \%$ ). But the pregnancy rates are higher than the general IVF population (57 versus $39.6 \%$ ). Of course these groups are not comparable in a lot of aspects, but it is an indication of the pregnancy outcome with IVF treatment after conservative surgery for deep endometriosis. These results are comparable as the results found by other authors [23, 24].

Conservative surgery for deep endometriosis in young women means preservation of organs, nerves, vascular blood supply and fertility. There is therefore a need for further strong and energetic debate to weigh up the benefits of shaving versus radical surgery. Feasibility is not always synonymous with efficacy.

Conflict of interest The authors report no conflicts of interest. The authors alone are responsible for the content and writing of the paper.

\section{References}

1. Koninckx PR, Meuleman C, Demeyere S, Lesaffre E, Cornillie FJ (1991) Suggestive evidence that pelvic endometriosis is a progressive disease, whereas deeply infiltrating endometriosis is associated with pelvic pain. Fertil Steril 55(4):759-765

2. Fauconnier A, Chapron C, Dubuisson JB, Vieira M, Dousset B, Breart G (2002) Relation between pain symptoms and the anatomic location of deep infiltrating endometriosis. Fertil Steril 78 (4):719-726 
3. Pouly JL, Drolet J, Canis M, Boughazine S, Mage G, Bruhat MA, Wattiez A (1996) Laparoscopic treatment of symptomatic endometriosis. Hum Reprod 11(Suppl 3):67-88

4. Macafee CH, Greer HL (1960) Intestinal endometriosis. A report of 29 cases and a survey of the literature. J Obstet Gynaecol Br Emp 67:539-555

5. Koninckx PR, Martin DC (1992) Deep endometriosis: a consequence of infiltration or retraction or possibly adenomyosis externa? Fertil Steril 58(5):924-928

6. Brouwer R, Woods RJ (2007) Rectal endometriosis: results of radical excision and review of published work. ANZ J Surg 77 (7):562-571. doi:10.1111/j.1445-2197.2007.04153.x

7. Chapron C, Chopin N, Borghese B, Foulot H, Dousset B, VacherLavenu MC, Vieira M, Hasan W, Bricou A (2006) Deeply infiltrating endometriosis: pathogenetic implications of the anatomical distribution. Hum Reprod 21(7):1839-1845. doi:10.1093/humrep/ de1079

8. Abrao MS, Podgaec S, Dias JA Jr, Averbach M, Silva LF, Marino de Carvalho F (2008) Endometriosis lesions that compromise the rectum deeper than the inner muscularis layer have more than $40 \%$ of the circumference of the rectum affected by the disease. J Minim Invasive Gynecol 15(3):280-285. doi:10.1016/j.jmig.2008.01.006

9. Payá V, Hidalgo-Mora JJ, Diaz-Garcia C, Pellicer A (2011) Surgical treatment of rectovaginal endometriosis with rectal involvement. Gynecol Surg 8(3):269-277. doi:10.1007/ s10397-011-0663-y

10. Dousset B, Leconte M, Borghese B, Millischer AE, Roseau G, Arkwright S, Chapron C (2010) Complete surgery for low rectal endometriosis: long-term results of a 100-case prospective study. Ann Surg 251(5):887-895. doi:10.1097/SLA.0b013e3181d9722d

11. Possover M, Diebolder H, Plaul K, Schneider A (2000) Laparascopically assisted vaginal resection of rectovaginal endometriosis. Obstet Gynecol 96(2):304-307

12. Keckstein J, Wiesinger H (2005) Deep endometriosis, including intestinal involvement-the interdisciplinary approach. Minim Invasive Ther Allied Technol 14(3):160-166. doi:10.1080/ 14017430510035916

13. Minelli L, Fanfani F, Fagotti A, Ruffo G, Ceccaroni M, Mereu L, Landi S, Pomini P, Scambia G (2009) Laparoscopic colorectal resection for bowel endometriosis: feasibility, complications, and clinical outcome. Arch Surg 144(3):234-239. doi:10.1001/ archsurg.2008.555, discussion 239
14. Donnez J, Squifflet J (2004) Laparoscopic excision of deep endometriosis. Obstet Gynecol Clin North Am 31(3):567-580. doi:10.1016/j.ogc.2004.06.006, ix

15. Roman H, Loisel C, Resch B, Tuech JJ, Hochain P, Leroi AM, Marpeau L (2010) Delayed functional outcomes associated with surgical management of deep rectovaginal endometriosis with rectal involvement: giving patients an informed choice. Hum Reprod 25(4):890-899. doi:10.1093/humrep/dep407

16. Kavallaris A, Köhler C, Kühne-Heid R, Schneider A (2003) Histopathological extent of rectal invasion by rectovaginal endometriosis. Hum Reprod 18(6):1323-1327

17. Remorgida V, Ragni N, Ferrero S, Anserini P, Torelli P, Fulcheri E (2005) The involvement of the interstitial Cajal cells and the enteric nervous system in bowel endometriosis. Hum Reprod 20 (1):264-271. doi:10.1093/humrep/deh568

18. Fanfani F, Fagotti A, Gagliardi ML, Ruffo G, Ceccaroni M, Scambia G, Minelli L (2010) Discoid or segmental rectosigmoid resection for deep infiltrating endometriosis: a case-control study. Fertil Steril 94 (2):444-449. doi:10.1016/j.fertnstert.2009.03.066

19. Anaf V, El Nakadi I, De Moor V, Coppens E, Zalcman M, Noel JC (2009) Anatomic significance of a positive barium enema in deep infiltrating endometriosis of the large bowel. World J Surg 33 (4):822-827. doi:10.1007/s00268-008-9903-3

20. Slack A, Child T, Lindsey I, Kennedy S, Cunningham C, Mortensen N, Koninckx P, McVeigh E (2007) Urological and colorectal complications following surgery for rectovaginal endometriosis. BJOG 114(10):1278-1282. doi:10.1111/j.1471-0528.2007.01477.x

21. De Cicco C, Corona R, Schonman R, Mailova K, Ussia A, Koninckx PR (2011) Bowel resection for deep endometriosis: a systematic review. BJOG 118(3):285-291. doi:10.1111/j.14710528.2010.02744.x

22. Mohr C, Nezhat FR, Nezhat CH, Seidman DS, Nezhat CR (2005) Fertility considerations in laparoscopic treatment of infiltrative bowel endometriosis. JSLS 9(1):16-24

23. Donnez J, Squifflet J (2010) Complications, pregnancy and recurrence in a prospective series of 500 patients operated on by the shaving technique for deep rectovaginal endometriotic nodules. Hum Reprod 25(8):1949-1958. doi:10.1093/humrep/deq135

24. Bianchi PH, Pereira RM, Zanatta A, Alegretti JR, Motta EL, Serafini PC (2009) Extensive excision of deep infiltrative endometriosis before in vitro fertilization significantly improves pregnancy rates. J Minim Invasive Gynecol 16(2):174-180. doi:10.1016/j.jmig.2008.12.009 